\title{
La Iglesia chilena desde 1973 a 1993: De buenos samaritanos, antiguos contrahentes y nuevos aliados. Un análisis politológico
}

\section{INTRODUCCIÓN}

"La parábola del Buen Samaritano define en términos sumamente concretos cuál debe ser la misión de la Iglesia en América Latina. La misión de la Iglesia significa siempre un servicio a los hombres, especialmente a hombres como el de la parábola: caídos y medio muertos (cfr. Lc. 10, 30). [...] Y la Iglesia encuentra el sentido de su existencia en ser la prolongación de este servicio de Jesús a todos los hombres, particularmente a los humillados y ofendidos de nuestra historia” (1).

La Iglesia Católica chilena en su compromiso por los derechos humanos durante el tiempo de la dictadura militar estuvo muy cerca del ideal que Leonardo Boff ilustra en esta cita. Su compromiso por los derechos humanos, la justicia social y la democracia juega un rol precursor en la Iglesia del continente latinoamericano. Este artículo pretende analizar el papel que tuvo la Iglesia Católica en el proceso político desde 1973 hasta 1993. No busca hacerlo desde la perspectiva de la teología o de la historia de la Iglesia, sino más bien haciendo uso de las herramientas que ofrece la ciencia política y la investigación empírica de procesos de transformación de sistemas políticos. El interés central es el de las posiciones y acciones políticas tomadas por la Iglesia Católica, sus orígenes y estrategias, y finalmente los efectos que ha tenido.

La primera parte (párrafo 2.) presenta algunos conceptos de la teoría política sobre la transición de sistemas autoritarios a la democracia. Estas herramientas sirven en la segunda parte (párrafos 3. a 5.) para analizar las posiciones y acciones políticas de la Iglesia Católica chilena.

\section{DESARROLLO DEL MARCO ANALÍTICO}

En la reflexión teórica sobre la transformación de sistemas políticos y procesos de democratización coexisten dos grandes posiciones: de una parte, aquellas posi-

(1) Boff 1986: 46 . 
ciones macroanalíticas, basadas en fundamentos estructuralistas, y de otra, las posturas microanalíticas (2). La discusión politológica, no obstante, demuestra que ambas posturas pueden ser vistas como complementarias. Una corriente que por el momento cuenta con gran acogida es la del Institucionalismo centrado en el Actor (Actor-Céntrico), en la cual se integran posiciones macro y microanalíticas (3). El Institucionalismo Actor-Céntrico parte de la premisa de que el marco de acción de los actores colectivos (así como también de los individuales) depende de los factores externos, que a su vez definen el contexto para la acción. Si bien el contexto no determina el actuar de los actores, lo impregna notablemente. De otra parte, el contexto no es algo rígido, como tampoco innamovible, sino que son los actores quienes lo construyen de manera activa. De esta manera queda claro que tanto el contexto como los actores tienen un papel central al pretender investigar los procesos políticos. Es por esta razón que en las siguientes reflexiones se definirá a la Iglesia como actor político, cuyo marco de acción se encuentra en el contexto de la dictadura y luego en el de la democratización.

\subsection{La Iglesia como actor político}

La Iglesia Católica -tanto la Iglesia en general, como también la Iglesia chilena- es desde muchos puntos de vista un actor político sui generis. A diferencia de otros actores no se legitima socialmente, funcionalmente o democráticamente, sino religiosamente (4). Su tarea y misión es anunciar el Evangelio del Reino de Dios. El servicio al hombre se orienta más que nada a la salvación escatológica del alma. Pero el hombre visto por la antropología cristiana es una unidad inseparable de alma y cuerpo. De allí que la salvación escatológica y el bien físico intramundano tampoco son separables. La Iglesia quiere servir a todo el hombre y por eso participa en el proceso político. De estos fundamentos teológicos del compromiso político de la Iglesia resulta que ella quiere, en primer lugar, lograr fines trascendentes. Paralelamente la Iglesia como organización, persigue metas muy concretas e immanentes: autoconservación, asegurar su posición dentro de la sociedad, proteger sus instituciones y sus bienes, la integridad física de sus miembros y funcionarios, mantener abiertos sus canales de influencia etc. Resulta obvio que la Iglesia se involucrará en los procesos políticos si se siente amenazada o si la realización de sus metas trascendentes o immanentes está en peligro.

Otros actores sociales importantes son los partidos políticos y los grupos de interés. La Iglesia sin embargo se distingue fundamentalmente de ellos: a diferencia de los partidos políticos, la Iglesia no quiere obtener responsabilidades gubernamentales. Ella no forma parte del sistema político y no trata de unificar intereses particulares de la sociedad, integrarlos y articularlos. Las posiciones que toma la Iglesia pretenden ser más bien intereses universales, válidos para toda la humanidad. A diferencia de los grupos de interés, la Iglesia no representa los intereses de sus

(2) Para una visión general veáse Merkel 1996.

(3) Cfr. por ejemplo Mayntz/Scharpf 1995 y Scharpf 1997.

(4) Cfr. Gaudium et Spes 76 y Ockenfels 1989: 6ss. 
miembros y no tiene su razón de ser en influir en el proceso político. La Iglesia entiende su compromiso intramundano e immanente en función de lo trascendente.

La Iglesia posee diversos canales de influencia en el ámbito político y social para defender sus intereses. Estos medios incluyen apelaciones a los gobernantes y a la opinión pública en forma de declaraciones y cartas pastorales. Otros medios son las acciones concretas y las relaciones de influencia de determinados funcionarios eclesiásticos. Como último medio está la posibilidad de la excomunión.

Otro instrumento de peso son los medios de comunicación eclesiásticos, los cuales bajo condiciones de censura no sufren una restricción tan extrema como los demás. A través de la difusión de informaciones sobre el país a nivel nacional e internacional por sus propios medios de comunicación, la Iglesia puede ejercer presión sobre el gobierno autoritario. Dadas las condiciones, la Iglesia puede contribuir a la movilización o desmovilización de la población. Por ser la Iglesia una institución bien definida en cuanto a sus recursos personales, materiales y de organización, puede jugar un rol central si la sociedad civil está desarticulada e inactiva (5).

Ahora bien, la pregunta que se presenta es ¿cuál es el alcance político de estos canales de influencia? El politólogo Hans-Joachim Lauth en su investigación sobre los sindicatos mexicanos expone el concepto bargaining power (poder de negociación) y distingue en él componentes políticos y económicos (6). En cuanto al aspecto económico, este concepto se refiere al potencial de influencia sobre los diferentes sectores del mercado. En el caso de la Iglesia chilena se puede constatar que este poder es de poca importancia. Aparte de los pocos empleados con los que la Iglesia cuenta, esta no juega un rol importante como empleador, inversionista o consumidor.

En cuanto al aspecto político entiende Lauth aquel poder de negociación que se basa en sus propios recursos y contribuye a asegurar el funcionamiento del proceso político (7). Entre los recursos propios de la Iglesia destaca especialmente su estructura estable, eficaz y casi 'invulnerable'. Tiene a su alcance varios medios de comunicación y canales seguros de información. Ella puede apoyarse en muchos militantes comprometidos y hasta cierto punto en sus miembros creyentes. Tampoco el peso social de la jeraquía y las élites religiosas debe dejarse de lado. En cuanto a recursos materiales, la Iglesia puede movilizar fuentes de financiamiento dentro y fuera del país

¿Pero cuáles son las funciones que brinda la Iglesia al sistema político? Aparte de su labor en el sector educativo y social, su función puede ser la de legitimar o deslegitimar el sistema político. A través de su influencia en la opinión pública, la Iglesia tiene un gran impacto en la aceptación que la población tiene de las políticas gubernamentales. En un caso como el chileno esta función legitimadora tiene un peso especial, porque el régimen muy a menudo intentó legitimarse a través de valores cristianos. Además del hecho de que muchos militares de la dictadura eran católicos (8). A este respecto, se puede afirmar que la Iglesia posee además una suerte de poder moral de negociación. A través de sus declaraciones la Iglesia

(5) Cfr. Lauth/Merkel 1997: 31 ss.

(6) Cfr. Lauth 1991: 42-50.

(7) Cfr. ibid.: 42.

(8) Cfr. Christi/Dawson 1996. 
presenta criterios para evaluar la política del gobierno. De esta manera intenta influir en el proceso político según sus propios intereses.

Aun a pesar de estos canales de influencia y del peso político que la Iglesia sin lugar a dudas tiene, se encuentra el dilema básico en el hecho de que la Iglesia tiene pocos medios eficaces para influir en la política. La raíz de este dilema está en el hecho de que la misión de la Iglesia no es de este mundo. Para tener éxito en sus intentos de influir a la política, la Iglesia necesita colaboradores y organismos que puedan aportar a sus ideas e intereses. Los más eficaces son aquellos colaboradores que por sí mismos poseen poder de negociación. Estos pueden ser actores de la sociedad civil o también partidos políticos. La Iglesia misma puede crear estos organismos de cooperación en el ámbito político. Un ejemplo es el catolicismo de asociaciones que está muy desarrollado en algunos países.

La Iglesia tiene un cuerpo doctrinario que le da base al contenido de sus declaraciones e intervenciones. Todo eso está vigente de manera permanente. Pero no siempre son los mismos aspectos los que destaca o por los cuales ella vela. Son dos los criterios decisivos sobre la posición que un tema tiene en la agenda pastoral y eclesial: Primero el contexto intra y extraeclesial y segundo las preferencias personales de los que toman las decisiones eclesiásticas. En la medida en que la Iglesia tiene que reaccionar a su entorno, ella pierde su autonomía para definir su agenda pastoral y eclesial. Las circunstancias externas pueden presionar a la Iglesia a realizar tareas que en el fondo no quiere asumir. Su acción se vuelve reactiva y ya no es proactiva. Se hace difícil el cumplimiento de las tareas meramente pastorales.

\subsection{El contexto social y político del actuar de la Iglesia}

La acción de la Iglesia se lleva a cabo en contextos concretos. El período analizado en este artículo empieza con el golpe de Estado en 1973, sigue con la institutionalización del sistema dictatorial y termina con la transición a la democracia y los primeros años de la consolidación hasta el año 1993. A continuación vamos a recapitular algunos aspectos centrales de la investigación politológica sobre autoritarismo y los procesos de transición democrática. Destacaremos las características de las distintas fases del proceso de democratización (9).

En cuanto al tipo de régimen, el Chile autoritario de los años 1973 hasta 1989 pertenece al subtipo del régimen burocrático-militar (10). El sistema político estaba marcado por un pragmatismo burocrático y militar y por una política represiva sin bases legales. Los militares trataron de legitimarse por valores como el patriotismo, la modernización y la doctrina de seguridad nacional. La gran mayoría de la población estaba excluida de la participación política. El control por parte del gobierno era extenso, pero no universal. Sobre todo los procesos económicos permanecieron fuera de la influencia del Estado. Siguendo el paradigma neoliberal y monetarista, el gobierno se limitó a crear el marco, para después dejar funcionar al mercado según sus propias leyes. Con las primeras jornadas de protestas en el año 1983 empezó el

(9) Cfr. Merkel 1999: 117-169, Merkel/Puhle 1999: 11ss.

(10) Cfr. Linz 1985. 
fin del régimen autoritario. Este quiebre del autoritarismo monolítico abrió el camino al llamado cambio de sistema (11).

El fin del régimen autoritario: Regímenes no democráticos suelen caer en el momento en el que ya no son capaces de legitimarse a largo plazo frente a una coalición de grupos sociales de peso (12). Este déficit de legitimación puede ser compensado por buenos resultados políticos o por represión. En el caso chileno se trata de una transición pactada, dirigida y controlada por las élites autoritarias. Esta etapa de apertura terminó con la derrota de Pinochet en el plebiscito del 1988.

Después de esta primera fase, vino la fase de la institucionalización de la democracia. Estuvo marcada por normas e instituciones inseguras. Las viejas normas e instituciones seguían en parte vigentes, mientras que las nuevas normas estaban desarrollándose, sin entrar aún en pleno vigor (13). Las viejas élites fueron poco a poco perdiendo el control. Al mismo tiempo que se establecían nuevas reglas institucionales (14). El anuncio y la preparación de elecciones libres -las llamadas founding elections - puso de manifiesto el inicio de esta nueva etapa de democratización (15). Se trató de un cambio de un poder anteriormente no limitado y controlado a un poder limitado y controlado, en el que el gobierno democrático cada ciertos años tiene que presentarse a la disposición de los electores. Bajo condiciones democráticas el proceso político está abierto a distintos resultados. En una dictadura los resultados son hasta cierto grado los que los gobernantes quieren lograr. Esta fase de construcción de las nuevas instituciones concluye por lo general con la aprobación de una nueva Constitución. De la inseguridad del proceso político se vuelve a la seguridad de procedimientos y normas constitucionales. El espacio de movilidad de los actores se hace más estrecho.

En el caso de Chile la derrota de Pinochet en el plebiscito del 1988 y el anuncio de las elecciones puede ser visto como el principio de la fase de la institucionalización. Como el fin de esta etapa, aquí no consideramos la tímida reforma constitucional de julio de 1989, sino las founding elections del 6 de diciembre de 1989.

Consolidación de la democracia: Con la asunción a la presidencia por parte de Aylwin empezó la difícil fase de la consolidación. Es importante tener en cuenta que -a diferencia de las fases anteriores- ya se trataba de un sistema democrático, un sistema esencialmente distinto al anterior. Los politólogos Brian H. Smith y Michael Fleet constatan en el caso chileno: "Differences have become matters of degree or detail, not principle, in many areas, and an atmosphere of mutual respect and accommodation has arisen" (16).

Aparte de la situación nacional, el contexto de la Iglesia mundial juega un rol importante para el hacer de una Iglesia nacional. Cabe mencionar aquí la integración en redes internacionales de solidaridad y de información. Pero también la

(11) Generalmente se distinguen tres fases del cambio de régimen: el fin del régimen autoritario, la institucionalización de la democracia y la consolidación de la democracia (cfr. Merkel 1999: 119169).

(12) Cfr. Merkel/Puhle 1999: 92ss.

(13) Cfr. Merkel 1999: 13ss.

(14) Cfr. Rüb 1994: 114s.

(15) Cfr. Merkel/Puhle 1999: 105

(16) Fleet/Smith 1996: 159. 
influencia del Vaticano sea por acciones directas o por su política de nombramiento de obispos.

\section{LA IGLESIA CATÓLICA Y LA DICTADURA EN CHILE}

Hay una diferencia entre lo que la Iglesia quiere o pretende ser y lo que de hecho es y hace. A continuación vamos a usar las herramientas anteriormente desarolladas para aplicarlas al caso concreto de la Iglesia chilena de los años 1973 a 1993. No se trata de una visión normativa o teologizante, sino más bien de un estudio empírico y analítico de su hacer concreto. El estudio se concentra en la Iglesia Católica chilena representada por su jeraquía y los organismos creados por ella (17).

Durante el régimen de la Unidad Popular la Iglesia trató de actuar como intermediaria entre las fuerzas políticas y sociales. Al llegar el 11 de septiembre, sin embargo, se vio justamente confrontada con la situación que ella había intentato evitar (18). Dos días después del golpe el Comité Permanente de la Conferencia Episcopal de Chile (CEC) por primera vez opinó respecto a la situación del país. Esta primera declaración estuvo marcada por la confianza en la integridad de los militares y el carácter transitorio de la intervención militar:

"Nos duele inmensamente y nos oprime la sangre que ha enrojecido nuestras calles, nuestras poblaciones y nuestras fábricas -sangre de civiles y sangre de soldados- y las lágrimas de tantas mujeres y niños. Pedimos respeto por los caídos en la lucha y, en primer lugar, por el que fue hasta el martes 11 de septiembre el Presidente de la República ( $\left.\mathrm{N}^{\circ} 2\right)$. Que se acabe el odio, que vuelva la hora de la reconciliación $\left(\mathrm{N}^{\circ} 3\right)$. Confiando en el patriotismo y desinterés que han expresado los que han asumido la difícil tarea de restaurar el orden institutional y la vida económica del país, tan gravemente alterados, pedimos a los chilenos que, dadas las actuales circunstancias, cooperen a llevar a cabo esta tarea, y sobre todo, con humildad y con fervor, pedimos a Dios que los ayude ( $\left.\mathrm{N}^{\circ} 5\right)$ " (19).

La gran mayoría de los obispos se abstuvo de comentar la situación. Pero seis de los cuarenta obispos se pronunciaron en favor del golpe (20). Entre ellos también Juan Francisco Fresno que más tarde iba a ser el sucesor del Cardenal Silva en la sede de Santiago. Pero también la oposición de Silva Henríquez contra el régimen al principio no estaba muy desarrollada: A los pocos días después del golpe, en un viaje por Europa expresó su lealtad a los militares:

(17) Para informaciones acerca de las iglesias evangélicas en Chile véase Sepúlveda 1999, Parker 1996: 70-112 y Ossa 1999; sobre la Iglesia popular durante el tiempo de la dictadura véase por ejemplo Pastor et al. 1993, Aldunate et al. 2000, Castillo 1986 y 1993 y la película documental ¡No podemos callar de lo que hemos visto y oído! Fragmentos de una Iglesia liberadora, Santiago 2000 .

(18) Para un breve sumario del rol de la Iglesia durante el tiempo de la Unidad Popular véase Fleet/ Smith 1996: 54-59.

(19) Declaración del Comité Permanente del Episcopado sobre la situación del país, 13 de septiembre de 1973, en Mons. Carlos Oviedo Cavada (ed), Documentos del Episcopado. Chile, 1970-1973. Santiago, Ediciones Mundo, 1974, 174.

(20) Cfr. Smith 1982: 292ss. 
"Como Cardenal, en nombre de la Iglesia, ofrecí al nuevo Gobierno de Chile la misma colaboración que la Iglesia había dado, en todas las obras del bien común, al Gobierno marxista del señor Allende. Al mismo tiempo, cosa que las autoridades aceptaron, he exigido la misma libertad de acción de que gozaba la Iglesia con el Gobierno precedente" (21).

Por medio de tales pronunciamientos la Iglesia dio cierta legitimidad moral al régimen militar.

Mientras la Iglesia se demoró meses en pronunciar su primera crítica pública al régimen, el perfil de una Iglesia samaritana se desarrolló pocos días después del golpe: El 9 de octubre 1973 fue fundado el Comité Pro Paz. Entre sus iniciadores estuvo el Cardenal Silva. El presidente del Comité fue el obispo luterano Helmut Frenz (22). Según sus estatutos, el Comité quería brindar apoyo económico, espiritual y jurídico a todos aquellos chilenos que se encontraban en la miseria personal o económica por la situación política del país. Temporalmente el Comité contó con unos 300 médicos, asistentes sociales y abogados en todo el país. Hasta su disolución a fines del año 1975 unas 7.000 personas buscaron apoyo jurídico, en los puestos de salud y en las clínicas unos 75.000 pacientes recibieron tratamiento médico. En los 400 comedores de todo el país muchos jóvenes y niños necesitados recibieron su comida diaria. Solo en Santiago se distribuyeron hasta 30.000 platos de comida por día. El Comité asistió a casi 5.000 extranjeros para salir del país. Se trataba en su mayoría de latinoamericanos de izquierda que por razones políticas habían dejado sus países y fueron acogidos por el Chile de Salvador Allende (23). Gracias al Comité llegó mucho apoyo económico del extranjero a Chile.

El primer paso hacia una denuncia profética por parte de la Iglesia demoró más de seis meses. En el documento La Reconciliación en Chile la CEC por primera vez criticó la situación que estaba viviendo el país:

"Nos preocupa, en primer lugar, un clima de inseguridad y de temor, cuya raíz creemos encontrarla en las delaciones, en los falsos rumores y en la falta de participación y de información.

Nos preocupan también las dimensiones sociales de la situación económica actual, entre las cuales se podrían señalar el aumento de la cesantía y los despidos arbitrarios o por razones ideológicas (...).

Nos preocupa, finalmente, en algunos casos, la falta de resguardos jurídicos eficaces para la seguridad personal que se traducen en detenciones arbitrarias o excesivamente prolongadas en que ni los afectados ni sus familiares saben los cargos concretos que las motivan; en interrogatorios con apremios físicos o morales; en limitación de las posibilidades de defensa jurídica (...).

Comprendemos que circunstancias particulares pueden justificar la suspensión transitoria del ejercicio de algunos derechos civiles. Pero hay derechos que tocan la dignidad misma de la persona humana, y ellos son absolutos e inviolables. La Iglesia debe ser la voz de todos y especialmente de los que no tienen voz" ( $\left.\mathrm{N}^{\circ} 5\right)$ (24).

(21) Citado en Fernández 1996: 135s.

(22) Cfr. acerca del Comité Lowden 1993: 189-203 y German 1999: 335ss.

(23) Cfr. Smith 1982: 313ss. y Canciono 1997: 30.

(24) Declaración del Episcopado de Chile "La reconciliación en Chile", en Mons. Carlos Oviedo Cavada (ed), Documentos del Episcopado. Chile, 1974-1980. Santiago, Ediciones Mundo, 1982, 16. 
"In erster Linie beunruhigt uns ein Klima der Unsicherheit und der Angst, deren Wurzeln wir in Denunziationen, in falschen Gerüchten und im Fehlen von Teilhabe und Information sehen. Ebenso beunruhigen uns die sozialen Auswirkungen der gegenwärtigen wirtschaftlichen Lage. [...] Schließlich beunruhigt uns in einigen Fällen das Fehlen wirksamen juristischen Schutzes für die persönliche Sicherheit, die sich ausdrückt in willkürlichen oder exzessiv ausgedehnten Festnahmen, bei denen weder die Betroffenen noch ihre Familien die konkreten Beschuldigungen kennen [...]; in Verhören mit physischem und moralischem Zwang, in der Beschränkung der Möglichkeit gerichtlicher Verteidigung; [...]. Wir verstehen, daß besondere Umstände die vorübergehende Aufhebung der Ausübung einiger bürgerlicher Rechte rechtfertigen können. Aber es gibt Rechte, die die Würde der menschlichen Person selbst betreffen, und diese sind absolut und unverletzbar. Die Kirche muß die Stimme aller sein, besonders derer, die keine Stimme haben".

A pesar de la crítica es notable un tono muy reservado. ¿Cómo es posible esta reserva por parte de los obispos? Muchos obispos vieron en la intervención militar el último remedio para evitar que el país se sumergiera en el caos (25). Eso también explica la opinión positiva que muchos de los obispos tenían de las Fuerzas Armadas. Pero después de medio año se hizo obvio que la intervención militar tenía un horizonte temporal más amplio. Los militares no querían dejar el poder en manos de los civiles. Anunciaron la elaboración de una nueva Constitución que iba a asegurar la posición privilegiada de las Fuerzas Armadas. A partir de eso la Iglesia empezó a criticar ya no solo sintomáticamente, sino que acusó a la política de los militares como causa de la miseria que el país y su población estaban viviendo.

El conflicto entre la Junta y la Iglesia se agudizó. Sobre todo la labor del Comité dio motivo para tensiones permanentes. Pinochet ordenó al Cardenal Silva disolver el Comité antes de que el gobierno lo hiciera. El Cardenal lo hizo a regañadientes. Pero pocas semanas después fundó la Vicaría de la Solidaridad. La Vicaría fue durante los siguientes años la punta de lanza eclesiástica contra la dictadura militar (26). De esta manera la Iglesia mostró que era capaz y que estaba dispuesta a institutionalizar su compromiso por los derechos humanos. Estaba preparada para exponerse a un conflicto duradero contra el régimen militar. Por falta de colaboradores seculares en esta situación de represión y de pluralismo limitado, la Iglesia creó su propio organismo para poner en práctica lo que estaba pronunciando en sus declaraciones. Creó la Vicaría con sus propios recursos. Las actividades de la Vicaría hablan por sí mismas: Hasta el fin de la dictadura la Vicaría ayudó a un promedio de 90.000 personas por año. Unos 11.000 recibieron asistencia jurídica. Entre 1973 y 1988 presentó -conjuntamente con su antecesor institucional- unos 9.000 cargos de violación de la Constitución, de los cuales sin embargo, apenas 23 fueron tratados jurídicamente. Gracias a la labor de la Vicaría no hubo en Chile casos de desaparecidos después de 1977. También el número de casos de tortura disminuyó notablemente. Por su táctica de juntar información, documentar y hacer públicos los casos de violaciones de derechos humanos, la Vicaría limitó el poder de los milita-

(25) Cfr. Smith 1982: 287

(26) Para la Vicaría véase por ejemplo Fruhling 1992, Precht 1995 y para la actividad publicista Pérez 1997. 
res (27). También contribuyeron las diversas acusaciones de los organismos de derechos humanos y la ONU y el aislamiento internacional. El poder de negociación de la Iglesia no se basó tanto en las apelaciones morales, sino más bien en el organismo poderoso que la Iglesia creó por sus propios esfuerzos y recursos. A través de este organismo la Iglesia hizo lo que estaba a su alcance para deslegitimar el régimen y aumentar el costo político de los militares.

El régimen por su parte optó por una estrategia ofensiva: tanto las instituciones de la Iglesia como sus empleados se convirtieron en objetivo permanente de las diversas campañas de los medios de comunicación y de la represión. Los conflictos aumentaron de tal manera que en el año 1980 la Iglesia por primera vez en un documento oficial habló de persecución contra la Iglesia (28).

Después del plebiscito exitoso del año 1980 la represión política disminuyó en algo. En este tiempo la oposición política empezó a organizarse. Durante la crisis económica de los años 1982/83 disminuyó el producto nacional en 15\%, mientras que la tasa de desempleo subió a más del 30\%. La mala ejecución económica del gobierno restó a los militares una importante base de legitimidad. Estos intentaron compensarlo aumentando la represión política. Pero la represión -debido a las actividades de la Vicaría- tuvo un costo político muy alto. El régimen no pudo evitar que los partidos políticos continuasen organizándose y que las redes sociales en los sectores populares se consolidaran.

Durante este tiempo se dio otro cambio importante en la postura de la Iglesia: Hasta el año 1982 las declaraciones episcopales se concentraban en los temas de los derechos humanos y la política económica. Pero con la carta pastoral El Renacer de Chile (17.12.1982) los obispos por primera vez exigieron la vuelta a la democracia. Para los años siguientes la demanda por la 'plena democracia' iba a ser el leitmotiv de las declaraciones eclesiales. Esta exigencia marca un cambio fundamental, en la medida en que la Iglesia ya no se limitaba solamente a criticar la política de los militares. Su crítica iba acompañada de un concepto alternativo al de las Fuerzas Armadas. En su demanda por la plena democracia, ella exigía la participación de los ciudadanos, el estado de derecho y la instauración de un acontecer político basado en la Constitución.

\section{LA IGLESIA CHILENA Y EL REGRESO A LA DEMOCRACIA}

Los regímenes autoritarios suelen caer cuando ya no pueden asegurar una base de legitimación a través de coaliciones con mucha influencia, represión o resultados políticos. La caída de la dictadura en Chile empezó lentamente con la crisis económica y la primera jornada de protestas del 11 de mayo de 1983. La sociedad desarticulada y muda volvió a la arena política. La prensa dejó de ser un instrumento usado por el régimen y desarrolló su función crítica. Los partidos políticos dejaron sus catacumbas y reconquistaron el espacio público. El clamor por la vuelta a la democracia se hizo cada vez más fuerte.

(27) Estas y otras estadísticas más se encuentran en los apéndices de Precht 1995: 53ss.

(28) Cfr. Carta a los Católicos de Chile: "Yo soy Jesús, a quien tú persigues" (29.5.80). 
Muchas fueron las víctimas de las jornadas de protesta. Más de cien los muertos. Muchos los heridos o detenidos (29). Los obispos llamaron a ambos lados a dejar la violencia y volver al diálogo. En sus declaraciones exigieron la libertad de información. Además, excomulgaron a todos los involucrados en la tortura (30). El hilo conductor de las posturas eclesiásticas era la demanda por la plena democracia.

Pero las apelaciones y llamados del episcopado tuvieron poco éxito. El régimen no les hizo caso. La que sí escuchó la voz de la Iglesia fue la oposición, que encontró en ella aspectos valiosos para su propia reflexión.

En el año 1983 ocurrió otro cambio importante en la Iglesia chilena: Por su edad el Cardenal Silva Henríquez dejó su cargo de Arzobispo de Santiago. Su sucesor fue Juan Francisco Fresno, cuyo nombramiento marcó un cambio de rumbo de la Iglesia chilena (31): Fresno -conocido por sus posturas teológicas y políticas bastante conservadoras- fue uno de los obispos que habían felicitado a Pinochet después del golpe (32). El régimen y las fuerzas conservadoras vieron este nombramiento con mucha satisfacción y alegría. Se hizo famoso el comentario de la esposa de Pinochet, cuando dijo a algunos periodistas que el Señor había escuchado sus oraciones (33). Pero el cambio de rumbo que temían los progresistas dentro de la Iglesia no se realizó. Fresno dejó bien claro que iba a seguir con la labor de la Vicaría de la Solidaridad como lo hacía su predecesor. Hacia adentro Fresno trató de disciplinar y despolitizar al clero. Las parroquias y los sacerdotes debían abstenerse de un compromiso político. Eso provocó muchas tensiones dentro de las CEBs. Algunos sacerdotes hasta dejaron su ministerio.

Frente a los militares Fresno trató de promocionar confianza: Retomó el cargo de gran canciller de la Universidad Católica, que su predecesor había abandonado al protestar contra la intervención de las Fuerzas Armadas en el ámbito universitario. Nombró a Raúl Hasbún como encargado de comunicaciones lo cual también fue una señal entendida por la Junta (34).

Pinochet apreció los esfuerzos hechos por Fresno. Se abrieron nuevos espacios de actuación para la Iglesia. Fresno fue invitado a facilitar el diálogo entre el ministro del Interior Jarpa y la joven Alianza Democrática (35). De la misma manera, Fresno trató de mediar en los conflictos durante las jornadas de protestas. Pero sus esfuerzos no fueron exitosos. El diálogo entre gobierno y oposición no tuvo lugar. Lo que sí puede ser visto como un éxito, fue el hecho que bajo el liderazgo de Fresno la Iglesia se convirtió en un interlocutor para dialogar y mediar. Cosa que pocos años antes había sido impensable. De este cambio se pudo aprovechar la Iglesia después de que se agotaran las protestas de 1985. Este agotamiento llegó a su punto final con el atentado infructuoso contra Pinochet en septiembre de 1986 y la

(29) Cfr. Huneeus 1985: 69 (cuadro 8) und 74 (cuadro 9).

(30) Así por ejemplo en Más allá de la protesta y la violencia en Iglesia de Santiago $\mathrm{N}^{\circ}$ 149, 7s., Un camino cristiano (15.12.83) o en Documentos del Episcopado 1988: 22-24.

(31) El nombramiento presenta un hito en la política vaticana de nombramientos episcopales, cfr. Ferari 1992.

(32) Cfr. Smith 1982: 292.

(33) Cfr. entre otros Cancino 1997: 150 y Meacham 1987: 412.

(34) Cfr. Meacham 1987: 415.

(35) Cfr. Guillaudat/Mouterde 1998: 158s. 
ola de represión política que le siguió. En esta situación de estancamiento político la Iglesia vio los 'Signos de los tiempos' y asumió un rol político activo: A partir de marzo de 1985 Fresno invitó a representantes de distintos partidos políticos a conversaciones particulares sobre la situación del país y estrategias para la vuelta a la democracia. A base de apuntes de estas conversaciones se elaboró un papel mostrando los puntos en común de los partidos políticos. En reuniones de representantes de los partidos políticos surgió el llamado Acuerdo Nacional para la transición a la plena Democracia. Los partidos exigieron la redemocratización pacífica, la normalización de la vida política, elecciones presidenciales directas y cambios de la Constitución (36). El régimen por su parte hizo lo posible por ignorar el documento y lo que estaba sucediendo en la sociedad civil. Aunque rechazado por el régimen, el Acuerdo Nacional representó un hito en el camino hacia la democracia. A la vez que fue uno de los éxitos más grandes de la Iglesia chilena. El Cardenal Fresno logró llevar la oposición desarticulada y dividida entre sí a un diálogo fructífero y a unir las fuerzas democráticas. Por otra parte, el Acuerdo Nacional ponía una vez en la discusión pública la pregunta sobre cuánto tiempo más iban los militares a mantenerse en el poder. Estos resultados fueron solamente posibles gracias a la iniciativa tomada por Fresno y gracias al espacio que él dio a las conversaciones políticas. En aquella situación Fresno fue el hombre adecuado en el momento justo. En vez de establecer el diálogo entre gobierno y oposición, realizó un acercamiento de las fuerzas democráticas, que al final llegaron a formar la Concertación de Partidos por la Democracia.

Otro acontecimiento importante fue la visita del Papa en 1987, la que fue preparada en un momento de violencia política en el país. Los militares trataron de usar la visita para sus propios fines. Lo lograron en parte. Desconociendo el protocolo, el Papa se presentó con Pinochet en el balcón de La Moneda. De otro lado, en una misa leyendo la Sagrada Escritura, Juan Pablo II lo hizo de la Biblia ensangrentada del P. André Jarlan, uno de los sacerdotes asesinados por los militares durante las jornadas de protestas. El Papa además visitó en el hospital a Carmen Gloria, una estudiante que durante una manifestación había sido rociada con gasolina y encendida por la policía (37). De manera general la visita del Papa se realizó sin grandes incidentes. La única excepción fue una misa celebrada por el Papa en el Parque O’Higgins. Durante la homilía se llevaron a cabo enfrentamientos violentos. Más de 600 personas fueron heridas y muchos manifestantes fueron detenidos (38). En conjunto, la visita papal fue un éxito para las fuerzas democráticas. Aparte del apoyo que expresó el Papa al compromiso de la Iglesia y de la reunión con representantes de los partidos políticos (incluyendo el PC) el gran evento de la visita fue que generó que el pueblo volviera a sentirse pueblo. Con casi dos millones de personas participando en los distintos actos, la visita papal fue la movilización más grande desde los tiempos de Allende.

(36) El documento fue publicado en Avetikan 1989: 362-366.

(37) Cfr. Klaiber 1996: 107s.; para un análisis de la visita del Papa veáse Lagos 1988: 257-272 y Weigel 2001: 530-536.

(38) Cfr. Guillaudat/Mouterde 1998: 177. 
De otra parte en 1987 los distintos partidos políticos fundaron el Comité de Partidos por las Elecciones Libres. De esta manera la oposición aceptó las reglas dadas por el mismo régimen en la Constitución de 1980: Esta preveía un plebiscito para el año 1988 para decidir si Pinochet debía seguir o no como presidente durante ocho años más. El PDC asumió un rol protagónico en la Campaña del NO. En muchas ocasiones los obispos llamaron a la inscripción en los registros. También animaron a los cuidadanos a votar sin miedo pero con conciencia (39). En su gran mayoría el episcopado no hizo públicas sus opiniones individuales. Solo tres obispos se pronunciaron a favor del NO (40).

Con la derrota de Pinochet en el plebiscito de 1988 terminó la primera fase de la redemocratización. El viejo régimen se iba acabando. Empezó la fase de la institucionalización de la democracia. En esta fase de normas inseguras la Iglesia se retiró de la vida política activa. En la medida de lo posible, los obsipos trataron de estabilizar la situación y de facilitar la transición a la democracia. La Conferencia Episcopal organizó reuniones informales entre gobierno y oposición para fomentar el diálogo (41).

Antes de las eleciones los obispos exigieron un voto consciente de los cuidadanos y una campaña que fuera orientada al bien común (42). En la declaración Por una transición en Paz (28.4.89) exigieron el respeto por la dignidad humana y por los derechos humanos. Se pronunciaron contra el odio y el revanchismo. Por primera vez se hacía mención de los temas del aborto y del divorcio (43).

Con la asunción al mando de Patricio Aylwin terminó la corta fase de la institucionalización de la democracia y empezó la difícil tarea de consolidar la democracia. Los militares usaron el interregno entre las elecciones y la asunción de Aylwin para asegurar su posición en el futuro Chile democrático. Con las llamadas Leyes de Amarre condicionaron a la democracia, la dejaron 'atada, pero bien atada'. De esta manera limitaron las posibilidades de acción de los gobiernos democráticos (44).

\section{LA IGLESIA Y LA DEMOCRACIA JOVEN}

Cuando Aylwin asumió la presidencia en marzo del 1990 era indudable que la Iglesia Católica era uno de los actores prodemocráticos clave durante el tiempo de la dictadura militar. Su compromiso político, social y pastoral salvó muchas vidas, alivió sufrimientos y dio consuelo. Su aporte a la rearticulación de la sociedad civil y de los partidos políticos fue central. Sorprendentemente casi todos los estudios

(39) Cfr. Declaración sobre la Inscripción en los Registros Electorales (10.7.87), en Documentos del Episcopado 1988: 235s., cfr. también Klaiber 1996: 109s. y Cancino 1997: 20s.

(40) Fueron los Obispos González, Hourton y Camus.

(41) Cfr. Klaiber 1996: 110.

(42) Cfr. por ejemplo: Al comenzar una nueva etapa (09.08.89), en Documentos del Episcopado 1992: 146ss.

(43) En Documentos del Episcopado 1992: 135ss.

(44) Cfr. German 1999: 387s. 
acerca del rol político de la Iglesia chilena abarcan solamente el tiempo de la dictadura (45). Pero vale la pena echar una mirada a la continudad y al cambio del compromiso político de la Iglesia Chilena durante el primer gobierno democrático.

La Iglesia sin lugar a dudas mantuvo sus posturas en los temas de los derechos humanos y de la justicia social. Siempre destacó la necesidad de aclarar las violaciones de los derechos humanos. También subrayó que para la reconciliación nacional era indispensable que los militares asumieran su responsabilidad política (46). En la declaración del Comité Permanente de la CEC El reencuentro que anhelamos a los veinte años del golpe, por primera vez se habló de una responsabilidad institucional por parte de las Fuerzas Armadas con respecto a las violaciones de los derechos humanos (47). Con sus llamados a aclarar las preguntas abiertas, con su compromiso por la reconciliación y el diálogo y con su opción por los pobres la Iglesia siguió en la línea que había tenido durante el régimen autoritario. En estos temas se mantuvo fiel a sus colaboradores de este tiempo. Siguió cooperando con los movimientos y organismos de la sociedad civil y los partidos políticos que formaban la Concertación.

Pero no es solamente continuidad. También hay cambios importantes, por no decir quiebres. Paradójicamente la Iglesia empezó a formar alianzas con aquellas fuerzas políticas que apoyaron a la dictadura militar y criticaron el compromiso de la Iglesia con los derechos humanos y la democracia. La Iglesia hizo uso de estas alianzas para discutir públicamente los 'temas nuevos', como por ejemplo el aborto, la educación sexual, el SIDA y sobre todo el tema del divorcio.

La declaración Certeza, coherencia y confianza. Mensaje a los católicos en una hora de transición (noviembre 1989) fue fundamental (48). Una vez más el documento toca los temas de la reconciliación, violaciones de los derechos humanos, injusticia económica, etc. Pero más allá de eso, el documento está marcado por los temores de los obispos que la nueva libertad política condujera a una liberación y erosión de las normas sociales y finalmente a una pérdida de valores cristianos. Este peligro se encontraba a sus ojos sobre todo en el ámbito de la moral individual, de la sexualidad y de la familia. A pesar de todas sus exhortaciones al diálogo, los obispos dejaron bien claro que el diálogo también tenía sus límites y que existen temas que no están para ser discutidos: "Una cosa es una legítima diversidad de opiniones en asuntos discutibles y otra cosa las divisiones y tensiones que hacen difícil el diálogo y a veces rompen la comunión 'afectiva y efectiva'" (49).

En este contexto, es sorprendente la vehemencia con la que los obispos trataron de bloquear cada intento por vestir de normas legales al divorcio (50). En

(45) Solamente Haas 1998, Fleet/Smith 1996 y Meacham 1994 incluyen en su análisis el rol de la Iglesia en la democracia joven.

(46) Así por ejemplo En una etapa nueva del país (27.4.90), en Documentos del Episcopado 1992: 174 ss. o El Reencuentro que anhelamos (8.9.93), en Pastoral Popular, № 233/Sept. 1993, 4-7, o los discursos de Oviedo, arzobispo de Santiago, en distintas ocasiones (cfr. Mensaje $\mathrm{N}^{\circ}$ 403, Oct. 1991, 414ss.).

(47) Cfr. Pastoral Popular, No 233/Sept. 1993, 4-7.

(48) Publicado entre otros en Persona y Sociedad, Vol. VI, No. 2-3/1990, 236-245 (= docla - Documentación social Católica latinoamericana, Año XVIII - julio-diciembre 1990).

(49) Ibíd., 237, lo subrayado es nuestro.

(50) Cfr. Haas 1998 a: 16s., Fleet/Smith 1996: 173s., un análisis profundo hace Blofield 2001. 
numerosas declaraciones los obispos se manifestaron abiertamente contra el divorcio. Para ellas recurrieron a argumentaciones del derecho natural, al carácter sacramental del matrimonio y la indisolubilidad del vínculo matrimonial. Además rechazaron el divorcio por razones sociales y funcionales (51). La declaración No separe el hombre lo que Dios ha unido (30.11.90) es fundamental para las siguientes declaraciones sobre este tema. Termina con una cita de Juan Pablo II:

“[...] No os [esposos y esposas, V.S.] dejéis invadir por el contagioso cáncer del divorcio, que destroza a la familia, esteriliza el amor y destruye la acción educativa de los padres cristianos. No separéis lo que Dios ha unido (cf. Mt 19, 6)" (52).

Así los obispos expresaron su rechazo categórico a cualquier legislación del divorcio. No se limitaron a apelar a sus fieles a no usar la posibilidad del divorcio, sino que además trataron de impedir que la posibilidad llegara a darse. Estas posturas inclinadas a una tutela paternalista difícilmente eran compatibles con el pensamiento liberal-democrático.

Con semejante afán los obispos se opusieron a los planes del gobierno de introducir educación sexual en los colegios. Tres fueron los argumentos lanzados por la Iglesia a este respecto: los programas de educación sexual transmiten valores erróneos y fomentan la promiscuidad sexual. La decisión sobre la introducción de estos programas no puede ser tomada democráticamente, porque decisiones sobre verdades morales no pueden ser tomadas democráticamente. Además -según los obispos- el Estado, al pretender asumir la educación sexual en los colegios, hace uso para ello de una política totalitaria (53).

En estos temas la Iglesia buscó el apoyo de los partidos derechistas y conservadores, y logró de esta manera bloquear las iniciativas de ley tanto en lo que respecta a la cuestión del divorcio como a la de la educación sexual. Estas posturas políticas se basaban en la convicción de que la legislación civil debe ser coherente con la legislación y doctrina moral de la Iglesia. Además de la cooperación de los partidos de derecha, la Iglesia usó la táctica de influir en la opinión pública y de ejercer presión a los mandatarios políticos. Insistió en que un político católico no puede aceptar una política que contradice a la enseñanza católica (54).

Parecía que la Iglesia tenía problemas para aceptar las consecuencias de los temas que ella misma había levantado. Fue difícil para la Iglesia aceptar el pluralismo democrático y la libertad tan anhelada en situaciones en que no vio representados sus intereses. Aceptó las reglas del juego solo mientras todo iba según sus intereses. Mientras que en los temas de los derechos humanos y la política social mantenía su postura progresista, se convirtió en otros campos en un actor de veto. Le costó aceptar que la 'voz de los sin voz' se convirtiera en una voz entre otras en el coro de lo social.

(51) Cfr. Unidos para siempre (22.07.91), en Documentos del Episcopado 1992: 226ss.

(52) En Documentos del Episcopado 1992: 198s., la cita está en la página 199. Cfr. también Por el bien de las familias en Chile. Documento oficial de la Asamblea Plenaria de la Conferencia Episcopal de Chile sobre el divorcio (22.04.94), en Pastoral Popular, Agosto 1994, 32.

(53) Cfr. Haas 1998: 52.

(54) Cfr. ibíd.: 57 y Blofield 2001: 23-27. 


\section{CONCLUSIONES}

Ha quedado demostrado que en el ámbito político la Iglesia actuó con mucha coherencia. En los años críticos de la dictadura militar, su compromiso por la democracia y los derechos humanos fue ejemplar, sobre todo porque entonces vio a menudo sus metas trascendentes e inmanentes en peligro. La Iglesia fue víctima de agresiones y hostigamientos. Por una parte, ella misma provocó este hostigamiento con sus acciones. Según el contexto la Iglesia empleó distintas estrategias: Durante la dictadura monolítica y la violencia sin límites, cuando no existían ni sociedad civil ni partidos políticos con quienes la Iglesia pudiera colaborar, ella creó su propio organismo de acción. El Comité pro Paz y la Vicaría fueron casi los únicos actores capaces de enfrentar el régimen. Este compromiso político la Iglesia lo pagó con la pérdida parcial de su autonomía al hacer su agenda. Para reaccionar a las exigencias que surgieron de la realidad vivida, ella hizo uso de una gran parte de sus recursos e influencias.

La Iglesia siguió con este rol durante la fase de la apertura del viejo régimen. Pero con el fortalecimiento de los partidos políticos y de la sociedad civil se retiró más y más de su rol activo. Gracias al cambio de Silva a Fresno se abrieron nuevos espacios, que la Iglesia supo usar, pudo así adaptarse al nuevo contexto y gracias a sus esfuerzos de mediación, sacar adelante el proceso de transición. La Iglesia fue exitosa porque una vez más asumió un rol protagónico, sin ser dependiente de la colaboración de los otros. En la fase de la institucionalización de la democracia se concentró en facilitar la transición y el diálogo entre los que en ese momento habían asumido el protagonismo del proceso de transición. De esta manera reconquistó su autonomía para establecer su agenda. Un indicio de ello es que durante esta fase se mencionan por primera vez otros temas propios de su agenda, como el divorcio por ejemplo.

De esta manera la Iglesia tuvo durante todos estos años éxito en la adaptación al contexto que ella misma había contribuido a crear. Durante los primeros tres años de la consolidación de la democracia las dificultades para adaptarse fueron mayores. No le resultaba fácil encontrar su lugar en un ambiente pluralista, liberal y democrático. Aprobó los procedimientos democráticos siempre y cuando no fueran divergentes con los intereses de la Iglesia. Para ello se encontró en la necesidad de buscar nuevos aliados, y los encontró en los partidos de derecha y conservadores, con sus dudosas posturas democráticas. La Iglesia se alió con fuerzas que un tiempo atrás habían sido sus adversarios políticos.

En cuanto a su poder de negociación es notable un desarrollo interesante que explica la búsqueda de nuevas alianzas: Una de las grandes ventajas de la Iglesia es su estabilidad como institución. Pero lo que durante la dictadura era una gran ventaja y excepción, bajo condiciones democráticas ya no tiene tanto peso. Durante la dictadura la Iglesia fue la voz más importante de la oposición. Pero en la democracia el número de los actores políticos y sociales ha aumentado notablemente. Además de que las posiciones de la Iglesia a favor de un Chile democrático contaban ya de por sí con un amplio apoyo en la arena política chilena. De otra parte, vale recalcar que muchas de las opiniones que la Iglesia defiende, son especialmente conflictivas. Mientras que durante la dictadura, con su pluralismo limitado y la 
ausencia de una sociedad civil, la Iglesia tuvo que crear sus propios organismos para aumentar su poder de negociación, ahora está en la situación de depender de sus colaboradores para realizar sus proyectos. Y finalmente la fuente más importante del poder de negociación de la Iglesia fue su poder moral. Su arma más fuerte fue la de deslegitimar el régimen militar. Un gobierno democrático en una sociedad pluralista no necesariamente depende del apoyo legitimador de la Iglesia. Ya que los gobiernos democráticos se legitiman gracias a procedimientos constitucionales y por resultados políticos.

\section{BIBLIOGRAFÍA}

Aldunate, José, et al., 2000: Crónicas de una Iglesia Liberadora, Santiago.

Arroyo, Gonzalo, 1975: Nota sobre la Iglesia y los Cristianos de Izquierda a la hora del Putsch en Chile, in: Latin American Perspectives, Vol. 2, Issue 1 (Spring 1975), pp. 88-99.

Avetikian, Tamara, 1989: Acuerdo Nacional y transición a la Democracia, en: Estudios Públicos, № 21 (verano 1989), pp. 309-387.

Blofield, Merike Helena, 2001: The politics of 'moral sin': a study of abortion and divorce in Catholic Chile since 1990, Santiago, Chile: FLACSO-Chile.

Boff, Leonardo, 1986: Teología desde el lugar del Pobre, Santander.

Cancino Troncoso, Hugo, 1997: Chile: Iglesia y dictadura 1973-1989. Un estudio sobre el rol político de la Iglesia Católica y el conflicto del régimen militar, Odense.

Castillo L., Fernando, 1993: Comunidades de Base: Fe y Política, en: Tópicos '90. cuadernos de estudio, 5/junio 1993, pp. 99-139.

Castillo, Fernando, 1986: Iglesia liberadora y política, Santiago.

Christi, Marcela/Dawson, Lorne L., 1996 : Civil Religion in Comparative Perspective: Chile under Pinochet (1973-1989), en: Social Compass 43 (3), 1996, pp. 319-338.

Documentos del Episcopado. Chile 1988-1991, Santiago 1992.

Documentos del Episcopado. Chile 1984-1987, Santiago 1988.

Ferari, José Manuel de, 1992: Diez años de cambios de obispos en Chile / 1981-1991, en: Reflexión y Liberación, III/12 1992, pp. 29-34.

Fernández Fernández, David, 1999: Oral History of the Chilean Movement 'Christians for Socialism', 1971-1973, in: Journal of Contemporary History, Vol. 34/2, pp. 283-294.

Fernández, David, 1996: La "Iglesia" que resistió a Pinochet. Historia, desde la fuente oral del Chile que no puede olvidarse, Madrid.

Fleet, Michael/Smith, Brian H., 1996: The Catholic Church and Democracy in Chile and Peru, Notre Dame.

Fruhling, Hugo, 1992: Resistance to Fear in Chile. The Experience of the Vicaría de la Solidaridad, en: J. Corradi/P. Weiss Fangen/M. A. Garretón (eds.): Fear at the Edge. State Terror and Resistance in Latin America, Oxford, pp. 121-141.

German, Christiano, 1999: Politik und Kirche in Lateinamerika. Zur Rolle der Bischofskonferenzen im Demokratisierungsprozeß Brasiliens und Chiles, Frankfurt a.M. 
Gill, Anthony, 1998: Rendering to Caesar. The Catholic Church and the State in Latin America, Chicago.

Guillaudat, Patrick/Mouterde, Pierre, 1998 Los movimientos sociales en Chile 1973-1993, Santiago.

Haas, Lies1, 1998: The Catholic Church in Chile: New Political Alliances, en: C. Smith/J. Prokopy (ed.): Latin American Religion in Motion: Tracking Innovation, Complexity and Change, New York. pp. 43-66.

Haas, Lies1, 1998a: The Effects of Institutional Structure on Policymaking: An Examination of Women's Rights Legislation in Chile 1990-1998. Paper delivered at the XXI. Conference of LASA, Sept. 1998, Chicago.

Huneeus M., Carlos, 1985: La política de la apertura y sus implicaciones para la inauguración de la democracia en Chile, en: Revista de Ciencia Política, Vol. VII, No. 1, pp. 25-84.

Klaiber S.J., Jeffrey, 1997: Iglesia, dictaduras y democracia en América Lati$n a$, Lima.

Lagos Schuffeneger, Humberto, 1988: Crisis de la esperanza. Religión y Autoritarismo en Chile, Santiago de Chile.

Lauth, Hans-Joachim, 1991: Mexiko zwischen traditioneller Herrschaft und Modernisierung. Die Gewerkschaften im Wandel von Politik und Wirtschaft (19641988), Münster/Hamburg.

Lauth, Hans-Joachim/Merkel, Wolfgang, 1997: Zivilgesellschaft und Transformation, en: H. lauth/W. Merkel (eds.): Zivilgesellschaft im Transformationsprozeß. Länderstudien zu Mittelost- und Südeuropa, Asien, Afrika, Lateinamerika und Nahost, Mainz, pp. 15-49.

Linz, Juan J., 1985: Art. Autoritäre Regime, en: D. Nohlen/R.-O. Schultze (eds.): Pipers Wörterbuch zur Politik, Bd. 1, Politikwissenschaft. Theorien - Methoden - Begriffe, München, pp. 62-65.

Lowden, Pamela, 1996: Moral Opposition to Authoritarian Rule in Chile, 1973-90, Houndmills/New York.

Lowden, Pamela, 1993: The Ecumentical Committee for Peace in Chile (19731975): Foundation of Moral Opposition to Authoritarian Rule in Chile, en: Bulletin of Latin American Research, Vol. 12, № 2, 1993, pp. 189-203.

Mayntz, Renate/Scharpf, Fritz W., 1995: Der Ansatz des akteurszentrierten Institutionalismus, en: R. Mayntz/F. Scharpf (eds.): Gesellschaftliche Selbstregelung und politische Steuerung, Frankfurt/New York, pp. 39-72.

Meacham, Carl E., 1994: The Role of the Chilean Catholic Church in the New Chilean Democracy, en: Journal of Church and State, Vol. 36/2, 1994, pp. 277-299.

Meacham, Carl E., 1987: Changing of the Guard: New Relationes between Church and State in Chile, en: Journal of Church and State, Vol. 29, 1987, pp. 411433.

Merkel, Wolfgang, 1999: Systemtransformation. Eine Einführung in die Theorie und Empirie der Transformationsforschung, Opladen.

Merkel, Wolfgang, 1996: Struktur oder Akteur, System oder Handlung: Gibt es einen Königsweg in der sozialwissenschaftlichen Transformationsforschung?, en: W. Merkel (ed.): Systemwechsel 1. Theorien, Ansätze und Konzeptionen, 2. ed., Opladen, pp. 303-332. 
Merkel, Wolfgang/Puhle, Hans-Jürgen, 1999: Von der Diktatur zur Demokratie. Transformationen, Erfolgsbedingungen, Entwicklungspfade, Opladen.

Molineaux, David J./Ress, Mary Judith, 1993: Maryknoll in Chile. The first fifty years, Santiago.

Noggler, Othmar, 1981: Chile, en: H.-J. Prien (ed.): Lateinamerika: Gesellschaft - Kirche - Theologie, Tomo 1, Aufbruch und Auseinandersetzung, mit Beiträgen von E. D. Dussel, O. Noggler, H.-J. Prien, Göttingen, pp. 220-273.

Ockenfels, Wolfgang, 1989: Zur sozialen und politischen Rolle der katholischen Kirche in der Bundesrepublik Deutschland, en: Aus Politik und Zeitgeschichte B 49/89, pp. 3-13.

Ossa, Manuel, 1999: Iglesias evangélicas y derechos humanos en tiempos de dictadura. La Confraternidad cristiana de Iglesia. 1981-1989, Santiago de Chile.

Parker Gumucio, Cristián, 1996: Las iglesias y su acción social en Chile, Santiago.

Parker, Cristián, 1990: El aporte de la Iglesia a la sociedad chilena bajo el régimen militar, en: Cuadernos Hispanoamericanos 482/483 (1990), pp. 31-48.

Pastor, Aníbal et al., 1993: De Lonquén a Los Andes. 20 años de Iglesia Católica Chilena, Santiago.

Pérez Mendoza, Consuelo, 1997: Los protagonistas de la prensa alternativa: Vicaría de la Solidaridad y Fundación de Ayuda Social de las Iglesias Cristianas, Santiago.

Precht Bañados, Cristian, 1995: En la Huella del Buen Samaritano. Breve Historia de la Vicaría de la Solidaridad, Santiago.

Rüb, Friedrich W., 1994: Die Herausbildung politischer Institutionen in Demokratisierungsprozessen, en: W. Merkel (ed.): Systemwechsel 1. Theorien, Ansätze und Konzeptionen, 2. ed., Opladen, pp. 111-137.

Scharpf, Fritz W., 1997: Games real actors play: actor-centered institutionalism in policy research, Boulder/Colorado.

Sepúlveda G., Juan, 1999: De Peregrinos a Ciudadanos. Breve Historia del Cristianismo Evangélico en Chile, Santiago de Chile.

Smith, Brian H., 1990: The Catholic Church and Politics in Chile, in: D. Keogh (ed.): Church and Politics in Latin America, Foreword by G. Green, Basingstoke, pp. 321-343.

Smith, Brian H., 1982: The Church and Politics in Chile. Challenges to Modern Catholicism, Princeton.

Stewart-Gambino, Hannah, 1992: Redefining the Changes and Politics in Chile, en: E. L. Cleary/H. Stewart-Gambino (eds.): Conflict and Competition. The Latin American Church in a Changing Environment, Boulder/London, pp. 21-44.

Weigel, George, 2001: Witness to Hope. The Biography of Pope John Paul II, New York.

Zorn, Reinhard, 1991: Iglesia y política económica en Chile. Un desafío para la evangelización 1973-1988, in: P. Hünermann (ed.): Enseñanza Social de la Iglesia en América Latina, Frankfurt, pp. 257-277. 


\title{
RESUMEN
}

El artículo analiza desde un punto de vista politológico el rol de la Iglesia católica chilena en los procesos políticos de los años 1973 a 1993. En un primer paso propone un marco analítico para el estudio de la Iglesia como actor político, poniendo énfasis tanto en el carácter de actor sui generis de la Iglesia como en el contexto sociopolítico donde se desarrolla la acción de la Iglesia.

En el segundo paso el artículo muestra el desarrollo paulatino y continuado de la oposición de la Iglesia a la dictadura: Desde la precaución de los comienzos hasta la ayuda sintomática a las víctimas, que fue acompañada por la denuncia de los abusos, la propuesta de alternativas al sistema y la mediación política activa. Así, la Iglesia se convirtió en uno de los actores políticos centrales de la redemocratización. Una vez recuperadas las condiciones democráticas, la Iglesia hizo nuevas alianzas con fuerzas reaccionarias y no democráticas para ver representados sus intereses en determinados campos de la política.

\begin{abstract}
The article analises, from a political perspective, the role of the Chilean Catholic Church in the political processes of the years 1973-1993. It first offers an analytic frame for the study of the Church as a political actor, emphasizing both its role as sui generis agent, and the sociopolitical context in which the Church operates. Secondly the article shows the gradual but constant development of the Church's opposition to the Dictatorship: From the caution of the beginnings to the overt help to the victims, together with the condemnation of the abuses, the proposal of alternative systems, and active political mediation. Thus the Church turned into one of the key agents of the re-democratization. Once the democratic conditions were recorvered, the Church created new partnerships with reactive and non-democratic forces to see its interests represented in certain fields of the politics.
\end{abstract}

